\title{
A simulation study of processes for mixing non-isothermal flows under dynamic effects
}

\author{
A.A. Sataev, V.V. Andreev, D.I. Novikov, Ju.S. Perevezentseva \\ sancho3685@mail.ru |vyach.andreev@mail.ru | grey1ngreen27@,gmail.com \\ Nizhny Novgorod state technical university n. a. R. E. Alekseev, Nizhniy Novgorod, Russia
}

\begin{abstract}
The processes for mixing of non-isothermal streams essentially define the parameters of the heat-carrier on an input in a core in modes with incomplete structure of the working equipment and, as a consequence, - a heat engineering condition of a core. Besides, the task of researching the temperature pulsations accompanying practically all modes of currents for non-isothermal streams is extremely relevant, as these pulsations lead to additional thermocyclic loadings on elements of the equipment and in many cases define its resource. The paper describes the research of mixing processes for non-isothermal water coolant flows in hydraulic model of ship nuclear power plant. In several experiments, attention was paid to the mixing processes when feeding non-isothermal flows through the circulation loops located opposite of each other. To simulate the effect of external dynamic force in the form of periodic effect on the spatial orientation of the model, the ship was tested on a stand "Swinging platform". These vibrations affected the mixing processes occurring within the model. The main impact they had on the transition time, temperature gradient, vertical component of the velocity projection. In the future, these parameters will be clarified and the influence of other factors on the mixing of non-isothermal flows in the ship's nuclear power plant will be studied in more detail.
\end{abstract}

Key words: non-isothermal stream, the ship's nuclear power plant, core simulator, the mixing.

\section{Introduction}

Reaction of the reactor core to perturbation of reactivity, which occurs due to a decrease in concentration of liquid absorber (boric acid) or temperature of coolant in one of loops of the first circuit essentially depends on degree of mixing with other unperturbed loops [1].

The work of nuclear power plant in transitional modes requirements on reliability of heat removal from reactor core and its safety should be fulfilled. The main transitional modes of operation of reactors of pressurized water reactor (PWR) type are operation at sudden decrease of the heat-carrier flow due to shutdown of the circulation pump of the 1st circuit and operation of nuclear power plant at resetting of electric load or shutdown of turbinegenerator [2].

Mixing processes are investigated by several scientific groups, including abroad. The result of their work were experimental installations: ROCOM (Germany), Vattenfall (USA), Fortum PTS (Finland), OKB "HYDROPRESS" (Russia).

Thus, at the HYDROPRESS stand the experiments were carried out on a four-loop model of the VVER-1000 reactor on a 1:5 scale [3].

It should be noted that most of the phenomena occurring in the systems under consideration can be studied with a sufficient degree of representativeness on small-scale models, from which a significant amount of information can be obtained [4].

All models involved mixing processes only in stationary modes on PWR-type reactor plants.

During operation the ship's reactor plant is subject to external dynamic forces of a different nature [5]. The ship is practically constantly under the influence of external dynamic forces influencing its spatial orientation both in normal operation and especially in emergency situations. In this paper, the effect of the periodic rocking phenomenon will be investigated.

The main impact of rocking in nuclear power plant is on the reactor and steam generator, as well as heat exchangers of mixing type, where non-isothermal mixing of flows takes place, which can negatively affect the operating modes of nuclear power plant. Also modern tendencies of nuclear power plant safety lead to the development of autonomous methods of chilling, natural circulation (NC) [6]. Due to the complexity of this process, which depends on many factors, the swinging also has a negative impact on it.

\section{Materials and methods}

Several small-scale models of the ship's nuclear power plant hydraulic path were used to study the processes of nonisothermal flows mixing. In [7-9] the mixing processes taking place in the single-network mixing model were described. It consists of one of four circulation loops. The nuclear power plant KLT-40 [10] (a shipboard nuclear power plant installed on a floating nuclear power plant ("Akademik Lomonosov"), as well as the icebreakers "Taimyr" and "Vaigach" and the light carrier ("Sevmorput") were taken as a prototype chosen for the modeling.

The circulation loop in this work is understood as a hydraulic path (from the entrance to the model (inlet nozzle)), a standpipe, a ring gap, a pressure collector, an imitator of the active zone (made in the form of hydraulic resistance - hole sheet (scale 1:5)) and an outlet nozzle.

The study of temperature distribution fields in characteristic areas of mixing was used as a main method of investigation. This is achieved by building a layer-bylayer picture of temperature distribution, which is done by changing the vertical coordinate of sensors located on the flow mixing path.

This work has identified the key factors that affect these processes. The influence of external dynamic force on the mixing of non-isothermal flows was also assessed.

However, this model required more detailed improvement and approaching to the real hydraulic path of the ship's nuclear power plant. Mixing issues, occurring during operation / disconnection of circulation loops, are also particularly interesting research topics.

To solve these problems, a four-loop stand was proposed to study the processes for mixing non-isothermal 
flows. The main technical characteristics of the stand are presented in table 1 .

Table 1. Main parameters of the experimental stand

\begin{tabular}{|c|c|}
\hline Parameter & Amount \\
\hline Simulated reactor plant & KLT-40 - 1:5 \\
\hline $\begin{array}{c}\text { Number of simulated circulation } \\
\text { loops }\end{array}$ & $19-38$ \\
\hline $\begin{array}{c}\text { The number of measuring probes in } \\
\text { the simulator }\end{array}$ & 6 \\
\hline $\begin{array}{c}\text { The number of probes for } \\
\text { monitoring the preparation of } \\
\text { injections of media }\end{array}$ & 4 \\
\hline Flow sensors & 3 \\
\hline Number of level sensors & 15 \\
\hline Reinforcement elements & $10-90^{\circ} \mathrm{C}$ \\
\hline Injection temperature &
\end{tabular}

The general view of the stand is shown in fig. 1, and the fuel assembly simulator is shown in fig. 2 .

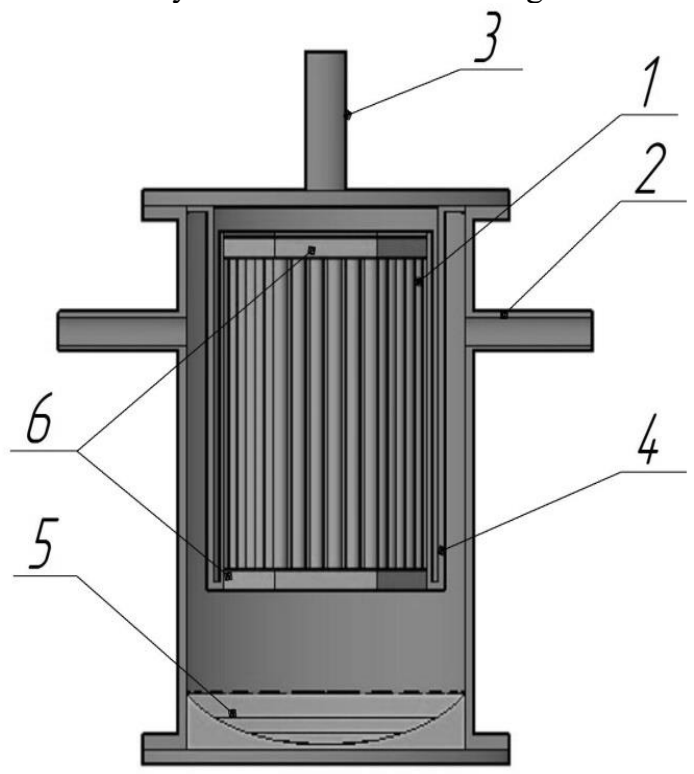

Fig. 1. General view of the research stand: 1 - fuel assembly simulator; 2 - pipe of the coolant inlet; 3 - pipe of the coolant outlet; 4 - shells; 5 - elliptical insert; 6 - remote grates)

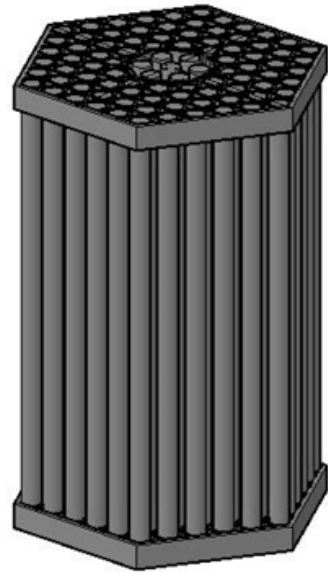

Fig. 2. Fuel assembly simulator

Similarity of processes in a natural reactor and in a model is observed under condition of geometrical, dynamic and thermal similarity. Similarity of heat transfer (as basic process in work) is observed under condition of equality of numbers Pekle $(\mathrm{Pe})$ in model and in natural object:

$$
\mathrm{Pe}=\frac{v \cdot l}{a}=\operatorname{Re} \cdot \operatorname{Pr}
$$

where $v$ - flow velocity; $l$ - characteristic size; a temperature diffusivity coefficient; Re - Reynolds number; Pr - Prandtl number.

The $\mathrm{Pe}_{\mathrm{mod}}$ number for our model is calculated:

$\mathrm{Pe}_{\text {mod }}=\mathrm{Re}_{\text {mod }} \cdot \operatorname{Pr}_{43} \approx 22 \cdot 10^{3} \cdot 4.07 \approx 8.95 \cdot 10^{4}$, (2) where the number Pr is taken for the average temperature in steady-state mode $\left(43^{\circ} \mathrm{C}\right)$.

Pe number for average coolant parameters in the core of KLT-40 reactor:

$\mathrm{Pe}_{\text {reac }}=\operatorname{Re}_{\mathrm{reac}} \cdot \operatorname{Pr}_{298} \approx 10^{5} \cdot 0.87 \approx 8.7 \cdot 10^{4}$, where number Re in the reactor is on average (1-5) $10^{5}$ $10^{7}$, number $\operatorname{Pr}$ is taken for average parameters of the coolant $\left(p=12.7 \mathrm{MPa} ; \mathrm{t}_{\text {average }}=298^{\circ} \mathrm{C}\right)$.

Accordingly, it is possible to draw a conclusion that the adopted model is applicable to the analysis of processes, as $\mathrm{Pe}_{\text {mod }} \approx \mathrm{Pe}_{\text {reac }}$.

A new core simulator has also been developed to provide a more detailed approximation of the simulated reactor plant. It is an equivalent cassette of KLT-40 main mass.

An equivalent cassette is a set of elements (simulator tubes) (shown in the fig. 3 and fig.4) of the main body of the fuel assembly mounted on the lower (support) and upper remote control grilles. Some of the rods (control rods) are placed behind the model cover through the grommets. Temperature parameters are controlled through these rods. Temperature sensors are located directly inside these channels (thin-walled channels are a sleeve). In addition, the cover is equipped with additional cable glands (in the course of this experiment are silenced) through which it is possible to install additional channels for measurement (temperature and pressure drop).

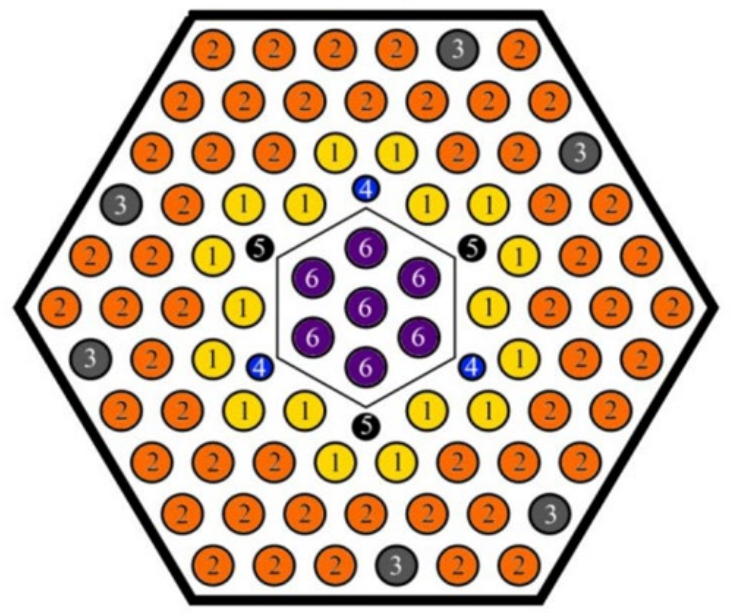

Fig. 3. Internal elements of the fuel assembly control unit 1 "light" fuel element (18), 2 - "heavy" fuel element (54), 3 burnable absorber 1 type (6), 4 - burnable absorber 2 type (3), 5 - zirconium displacer (3), 6 - compensating rods (6) 


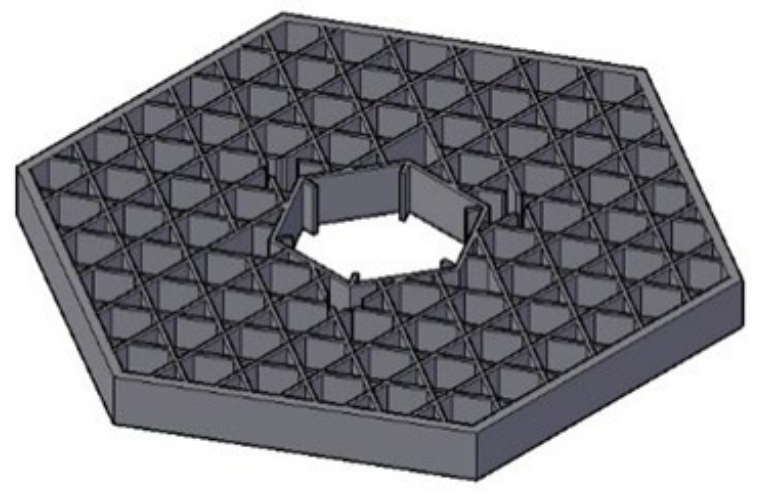

Fig. 4. General view of the remote gratings

To model the impact of external dynamic force in the form of periodic impact on the spatial orientation of the model a "Swinging platform" stand was proposed (fig.5). It consists of a frame rigidly fixed to the power plate. The Oscillating platform is connected to the frame through the shaft and bearings. The electric motor through the crank mechanism transmits a rotational force on a rod which in turn drives the investigated model on a platform. Due to the change of the crank radius as well as the frequency of rotation of the electric motor (frequency converter), it is possible to change the amplitude and period of the rocking model.

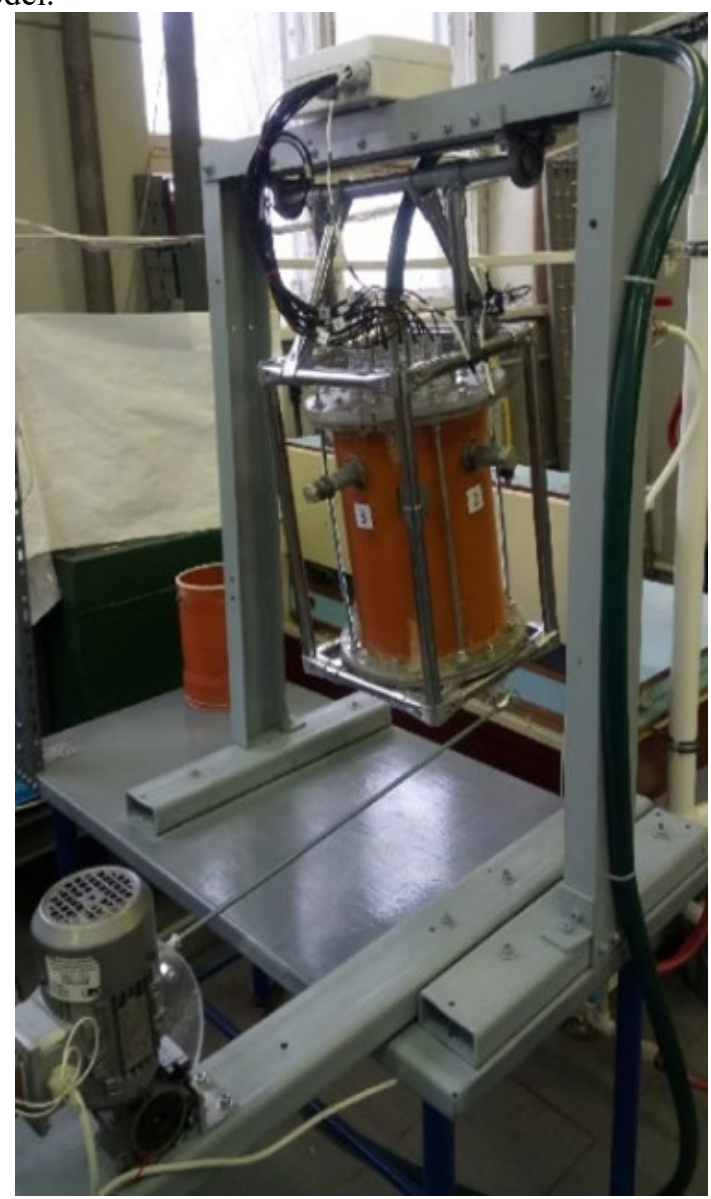

Fig. 5. General view of the "Swinging platform" stand

The model can vibrate in a given plane, like a mathematical pendulum, by law:

$$
\phi=\phi_{\max } \cdot \sin \left(\frac{2 \pi \tau}{T}\right),
$$

where $\varphi$ - corner, $\varphi_{\max }-$ oscillation amplitude, [Rad]; T - the oscillation period, [s]; $\tau$ - the current time, [s].

In this work, the media were supplied to the model from two spigots (1 and 3) located opposite each other.

In the dynamic mode, the flow was fed simultaneously with the harmonic oscillations of the model with an amplitude of $15^{\circ}$ degrees and a period of 4 seconds.

\section{Results}

The diagrams (top view) of temperature distribution through control channels are shown below (figs. 6. and 7.). Temperature sensors are located at the bottom at the input to the model-simulator core (except for sensors of channels 18 and 78, where sensors are also located at the output of the simulator). The central hexagonal channel for compensation rods is plugged.

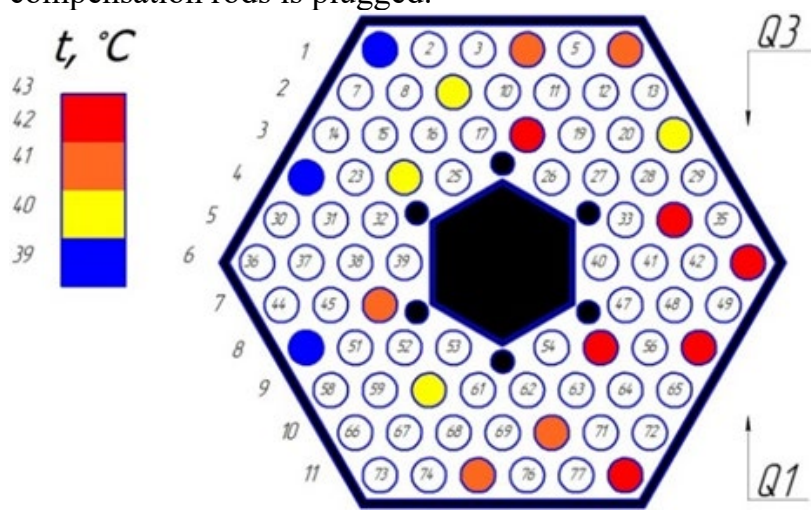

Fig. 6. Input temperature distribution of to the simulator active zone in a stationary mode

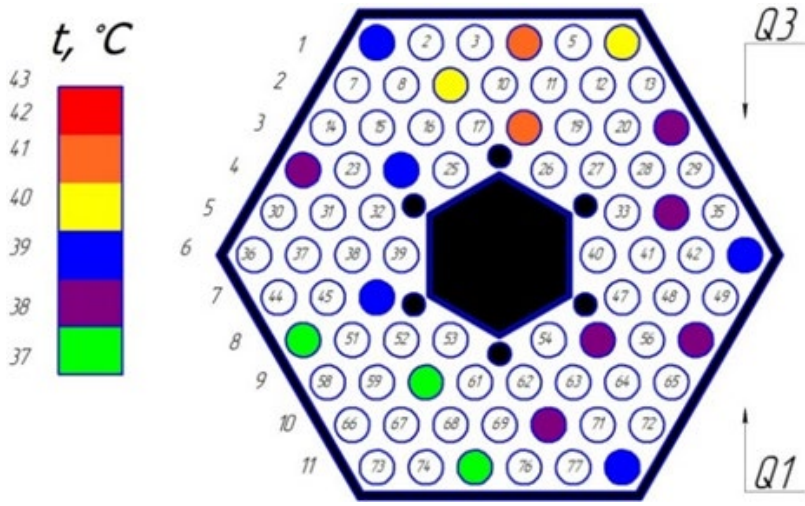

Fig. 7. Distribution of temperature at the input to the simulator active zone in dynamic mode

The data obtained in the course of the work have been processed and the key points are given in Table 2.

Table 2. Border conditions

\begin{tabular}{|c|c|c|}
\hline Parameter & $\begin{array}{c}\text { Fixed } \\
\text { position }\end{array}$ & Swinging \\
\hline $\begin{array}{c}\text { Volume flow } \\
(\mathrm{Q} 1) / \text { temperature }(\mathrm{t} 1)\end{array}$ & $\begin{array}{c}10 \mathrm{l} / \mathrm{min} / \\
60^{\circ} \mathrm{C}\end{array}$ & $\begin{array}{c}10 \mathrm{l} / \mathrm{min} / \\
60^{\circ} \mathrm{C}\end{array}$ \\
\hline $\begin{array}{c}\text { Volume flow } \\
\text { (Q3)/temperature (t3) }\end{array}$ & $\begin{array}{c}10 \mathrm{l} / \mathrm{min} / \\
20^{\circ} \mathrm{C}\end{array}$ & $\begin{array}{c}10 \mathrm{l} / \mathrm{min} / \\
20^{\circ} \mathrm{C}\end{array}$ \\
\hline $\begin{array}{l}\operatorname{Re} 1 / \operatorname{Re} 3 \text { (on input to the } \\
\text { model) }\end{array}$ & $22367 / 10574$ & $22367 / 10574$ \\
\hline Swing amplitude & - & $15^{0}$ \\
\hline Swing period & - & $4 \mathrm{sec}$. \\
\hline Time of transition & $\approx 225 \mathrm{sec}$. & $\approx 174 \mathrm{sec}$. \\
\hline
\end{tabular}




\begin{tabular}{|c|c|c|}
\hline $\begin{array}{c}\text { Vertical projection of } \\
\text { velocity } \Delta \mathrm{z} / \Delta \tau(\text { on } \\
\text { channel } 78), \text { where } \\
\Delta \mathrm{z}=\mathrm{f}(\Delta \mathrm{t})\end{array}$ & $\approx 14 \mathrm{~cm} / \mathrm{s}$ & $\approx 3.5 \mathrm{~cm} / \mathrm{s}$ \\
\hline $\begin{array}{c}\text { Vertical projection of } \\
\text { velocity } \Delta \mathrm{z} / \Delta \tau \text { (on } \\
\text { channel } 18), \text { where } \\
\Delta \mathrm{z}=\mathrm{f}(\Delta \mathrm{t})\end{array}$ & $\approx 17.5 \mathrm{~cm} / \mathrm{s}$ & $\approx 3 \mathrm{~cm} / \mathrm{s}$ \\
\hline $\begin{array}{c}\text { Maximum temperature } \\
\text { gradient in steady-state } \\
\text { mode }\end{array}$ & $4{ }^{0} \mathrm{C}$ & $3.82{ }^{\circ} \mathrm{C}$ \\
\hline
\end{tabular}

\section{Discussion}

Having analyzed the results obtained, the following conclusions can be drawn:

4.1. In stationary operation (without jogging), the hot flow is counterclockwise, cold flow is in a clockwise direction. The maximum temperature is concentrated in the right side sectors (near the wall) for both cold and hot flow.

During the work analysis [11] where authors used a technique of research of mixing of streams by conductometric method, at similar modes of work in the field of natural circulation of the heat-carrier at giving in circulation loops of solutions with different salt content the following laws correlating with our results have been revealed:

4.2. On the periphery there are two segments with "zero" trace concentration, where there was no mixing.

4.3. According to the location of these segments and the place of formation of the front kernel with an increased concentration of the tracer ("heavy" salt solution - if we interpret it by the method described above - cold flow Q3), we can see that there was a clockwise twist of the flow.

4.4. In dynamic mode, the flow curl is disturbed. This is probably due to the fact that the dynamic force acting in the direction parallel to the spigots moves the hot vortex to the opposite wall. The cold vortex also shifts, but the degree of its displacement is greater. This is due to the fact that the centrifugal force has a greater influence on the cold vortex (due to its higher density and viscosity) than on the hot vortex.

This confirms the mathematical model described by us in [9], where the centrifugal force was set when exposed to an elementary mole as a ratio:

$$
\partial F_{c}=\rho R\left(\frac{\partial \phi}{\partial \tau}\right)^{2} \partial v
$$

where, $\partial \mathrm{v}$ - volume change, $\left[\mathrm{m}^{3}\right] ; \rho$ - density, $\left[\mathrm{kg} / \mathrm{m}^{3}\right] ; \mathrm{g}$ free fall acceleration, $\left[\mathrm{m} / \mathrm{s}^{2}\right] ; \mathrm{R}$ - radius, $[\mathrm{m}] ; \partial \varphi$ - angle element, [rad]; $\partial \tau$ - elementary time increment, [s].

From this dependence it follows that the degree of influence on the external dynamic force flow is directly proportional to the density of the coolant.

4.5. The transition time of the rocking action is reduced

4.6. The vertical projection of speed of a stream calculated indirectly on change of temperature, makes for a stationary mode on the average $\approx 15-16 \mathrm{~cm} / \mathrm{s}$ that is comparable to speed of natural circulation of the heatcarrier $(10-20 \mathrm{~cm} / \mathrm{s})$. In dynamic mode, the vertical component is much smaller (about $3 \mathrm{~cm} / \mathrm{s}$ ). It means that the stream influencing external force receives acceleration along a rolling plane, that is the horizontal projection of a vector of speed of a stream increases.

4.7. The maximum temperature gradient when exposed to external dynamic force decreases slightly.

\section{Conclusion}

Research of processes for mixing non-isothermal water coolant flows in a hydraulic model of ship nuclear power plant KLT-40 has been carried out. In the experiments, attention was paid to the mixing processes when feeding the non-isothermal flows through the circulation loops located opposite each other. The results correlated with the results of other leading scientific groups dealing with this problem and used other methods that have been obtained.

To investigate the effect of external dynamic forces, this model was tested under the effect of periodic oscillations. These oscillations influenced the mixing processes occurring within the model. The main impact they had was on the time of transition, temperature gradient, vertical component of the velocity projection. In the future, these parameters will be clarified and the influence of other factors on the mixing of non-isothermal flows in the ship's nuclear power plant will be investigated in more detail.

\section{Acknowledgments}

The paper was performed with the support by RFBR, Grant № 19-07-00455.

\section{References}

[1] Prasser H-M, Grunwald G, Höhne T, Kliem S, Rohde U and Weiss F-P 2003 Coolant Mixing in a Pressurized Water Reactor: Deboration Transients, Steam-Line Breaks, and Emergency Core Cooling Injection Nuclear Technology 143 1 37-56.

[2] Sarkisov A A 1964 Dynamics of nuclear power plants of submarines (Sevastopol: Sevastopol Higher Naval School).

[3] Lisenkov E A, Bezrukov Yu A, Ulyanovsk V N, Saliy L A, Ulyanovsk D V, Zaitsev D V, Sergeev S G, Bykov M A and Zaitsev S I 2008 Research of mixing of the heat carrier in the lower chamber of the reactor Questions of nuclear science and technology Series: «Ensuring the safety of nuclear power plants» 233 17.

[4] Krapivtsev V G and Solonin V I 2019 Model studies of inter-loop mixing heat carrier in the VVER-1000 in reactor pressure path Atomic energy 125 271-77.

[5] Kudinovich I V 2019 Justification of the nuclear and radiation safety of an atomic vessel under external influences Proceedings of the Krylov State Scientific Center 1 (387) 131-42.

[6] Blagoveshchensky A Ya, Bor S M and Mityukov V N 2015 Ship nuclear energy technologies in solving the problems of reliability, safety and survivability of Russian NPPs Sci. and Tech. Coll. Life Cycle Support Technologies for NPPs (Sosnovy Bor: NITI im. A.P. Alexandrova) pp 24-37.

[7] Sataev A A, Duntsev A V, Vorobev D A and Krasavin N A 2018 Study of mixing processes of nonisothermal 
flows on a single-loop model of a reactor installation Modern high technology 3 96-101.

[8] Sataev A A and Duntsev A V 2018 Investigation of the processes of mixing non-isothermal flows on a model of a ship's nuclear power plant Bulletin of ISEU 5 26-32.

[9] Berezin A A, Sataev A A, Khvoinov O V and Duntsev A V 2019 Assessment of the influence of an external dynamic force on the mixing of non-isothermal flows in the reactor plant Bulletin of ISEU 5 5-11.

[10]Advances in small modular reactor technology developments. A Supplement to: IAEA Advanced Reactors Information System (ARIS) 2018 (Austria: IAEA).

[11]Zaitsev D V, Lisenkov E A, Ulyanovsky D V and Bezrukov Yu A 2011 Investigation of mixing of coolant in a reactor with two circulation loops Proc. of the 7th Int. sci. and tech. Conf. Ensuring the safety of nuclear power plants with VVER (Podolsk: OKB «GIDROPRESS»).

\section{About the authors}

Sataev Alexander A., postgraduate student of the Department «Nuclear reactors and power plants», Nizhny Novgorod state technical university n. a. R. E. Alekseev. E-mail: sancho3685@mail.ru

Andreev Vyacheslav V., Head of the Department «Nuclear reactors and power plants», Grand $\mathrm{PhD}$ of Sciences in technology, associate professor, Nizhny Novgorod state technical university n. a. R. E. Alekseev. E-mail: vyach.andreev@mail.ru

Novikov Denis I., master's degree student of the Department «Nuclear reactors and power plants», Nizhny Novgorod state technical university n. a. R. E. Alekseev. E-mail: grey1ngreen27@gmail.com

Perevezentseva Iuliia S., Associate of Professor of the Chair of Foreign Languages, Ph.D., Nizhny Novgorod state technical university n. a. R. E. Alekseev. E-mail: khohlova@pochta.ru 